\title{
An Adaptive Spacing Policy Guaranteeing String Stability in Multi-Brand Ad Hoc Platoons
}

\author{
Gábor Rödönyi, Member, IEEE
}

\begin{abstract}
A method is presented for the longitudinal control of autonomous vehicles forming a multi-brand, ad hoc platoon. A leader and predecessor following (LPF) control architecture is known to allow string stable platooning with shorter safety gaps between vehicles as compared with predecessor following schemes. General LPF strategies, however, require the exact knowledge of spacing policies of predecessor vehicles for correctly specifying a spacing with respect to the leader. It follows that arbitrary spacing policies in ad hoc platoons prevent the applicability of classical LPF control structures. It is shown in this paper that it is possible to exploit the advantages of LPF architectures in multi-brand platoons without a priory knowledge of spacing policies of predecessors. The unknown spacing policies are replaced by a virtual one, which serves as an input to a two degree of freedom LPF controller. The resulting control structure enables the organization of ad hoc platoons consisting of vehicles with different spacing policies. Computer simulations are presented to illustrate the statements.
\end{abstract}

Index Terms-Multi-brand platoons, ad hoc platoons, heterogenous platoons, string stability, adaptive spacing policy.

\section{INTRODUCTION}

$\mathbf{P}$ LATOONS consist of a number of automated vehicles, one closely following the other. They are constructed for advancing increased road capacity, reduced fuel consumption, and improved safety. Platoons are characterized, among other features, by control architecture and the type of spacing policy [1]. The most common control architectures are leader and predecessor following (LPF) and predecessor following (PF) scheme, the most common spacing policies are constant (CSP) and constant time-headway (CTHSP) spacing policy. An important property of vehicle strings, either driven by humans or autonomously, is string stability [2], [3], the transient property of the string. The lack of string stability may cause traffic jams [4]. Information from the leader vehicle in LPF architectures allows for short, constant spacing and string stability, while string stability with PF control architectures can be achieved only by much longer, speed dependent spacing [5].

At the current state of developments, it is common to assume that all vehicles in a platoon share the same control architecture and the same spacing policy. Two facts motivate the relaxation of these assumptions. On the one hand, there

This work was supported by the János Bolyai Research Scholarship of the Hungarian Academy of Sciences and by the National Research Development and Innovation Fund through the project "SEPPAC: Safety and Economic Platform for Partially Automated Commercial vehicles" (VKSZ 14-1-2015-0125). G. Rödönyi was with Systems and Control Laboratory, Institute for Computer Science and Control, Hungarian Academy of Sciences. rodonyiesztaki.hu

Accepted, final version

(C) 2017 IEEE Transactions on Intelligent Transportation Systems

Digital Object Identifier 10.1109/TITS.2017.2749607 is a need for developing solutions for multi-brand platooning so that platooning technology can better proliferate [6]. The above assumptions imply restrictions on the vehicles that can join a particular platoon, practically prohibiting the organization of general multi-brand platoons. Without the assumptions, each manufacturer could develop its own preferred control strategy and spacing policy, yet the vehicles could efficiently cooperate with each other. On the other hand, the degree of autonomy in vehicle driving and the degree of cooperation between vehicles and roadside infrastructure are increasing thanks to the efforts made in the field of intelligent transportation systems [7]. Adaptive cruise control (ACC) is already available in more and more cars, while cooperative adaptive cruise control (CACC) built on vehicle to vehicle (V2V) communication technologies is expected in the next decades [8]-[11]. The rising number of these vehicles with car following functionalities implies the increasing probability of their meeting and forming unintended, unorganized, ad hoc platoons. Thus any vehicle following method should guarantee string stability and good tracking performance also in arbitrary diverse and heterogeneous ad hoc platoons.

This paper is motivated by the spacing problem caused by the diversity of spacing policies in a platoon. We focus on the phenomena arising when the control of a vehicle utilizes relative position information from multiple predecessor vehicles. An example is the classical LPF control structure commonly applied in well organized, fully automated platoons where reference positions with respect to both the leader and the direct predecessor are defined in a consistent way [3]. When an LPF control architecture is applied with position feedforward in arbitrary ad hoc platoons, where predecessors follow unknown spacing policies, collisions may occur [12].

Closely related problem was discussed in [13], [14], where the heterogeneous platoon consisted of mixed human driven and cooperating autonomous vehicles, but the above problem was circumvented in [14] by communicating only acceleration measurements between distant vehicles, and so prescribed spacing for a specific vehicle was defined only with respect to the direct predecessor vehicle. AnnieWAY, the winner team in the 2011 Grand Cooperative Driving Challenge chose a control strategy where the reference position and the corresponding control action were computed with respect to each predecessor vehicles, then the smallest control action was selected [15]. The strategy worked safely in a short multivendor platoon, but string stability and scalability of the concept was not considered. At the same competition, the authors in [16] applied a string stable CACC design with acceleration feedforward from the leader. In this way, reference position was defined 
only with respect to the direct predecessor.

It is shown in the following sections that efficient controllers of more general structure (with feedback of position and speed information from distant vehicles) can also be applied in a heterogeneous, multi-vendor platoon, provided that an appropriate adaptive spacing policy (ASP) is used. In the proposed LPF-ASP control structure, the advantages of classical LPF architectures (short spacing) and PF architectures (no need for agreement in spacing policies) are combined without their disadvantages (need for synchronization and long speed dependent spacings, respectively). Preliminary results can be found in [12], where, in contrast to the present paper, string stability was not elaborated. A simplified LPF-ASP control structure was developed in [17] to handle spacing problems caused by actuator saturations in predecessor vehicles.

The presented approach for examining the transient properties of the string exploits the unidirectionality of the information flow topology. In these networks, stability is guaranteed by the stability of the components. In general interconnection structures with bidirectional information flow, however, stability, and scalability of stability are important issues that can be studied by, e.g., graph theoretic methods [18], [19].

With respect to the existing literature on ad hoc platooning, the contribution of the present paper can be summarized in the following four features. 1) The proposed control architecture utilizes leader's position information to improve spacing performance; 2) No agreement in spacing policies among the vehicles is necessary; 3) String stability is guaranteed; 4) general theoretical results on heterogeneous string stability are derived in order to evaluate the proposed method. We restrict our attention to linear time-invariant systems and we focus only on the basic concept. Effects of disturbances, sensor noise, delays and nonzero initial conditions are not considered here.

The basic problem is introduced in Section II. The proposed spacing policy adaptation method is presented in Section III. Conditions for heterogeneous string stability and design considerations are provided in Sections IV and V. Numerical analysis and simulation results are discussed in Section VI.

Notations. For $p \in[1, \infty]$ the function space $\mathcal{L}_{p}$ denotes $\left\{x:[0, \infty) \mapsto \mathbb{R}^{n}: x\right.$ is measurable and $\left.\|x\|_{p}<\infty\right\}$, where $\|x\|_{p} \triangleq\left(\int_{0}^{\infty}|x(t)|^{p} d t\right)^{1 / p}$ for $p \in[1, \infty)$ and $\|x\|_{\infty} \triangleq$

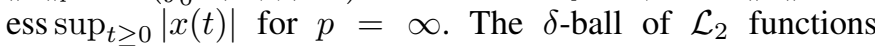
is denoted by $\mathcal{B L}_{2}(\delta) \triangleq\left\{x \in \mathcal{L}_{2}:\|x\|_{2}<\delta\right\}$. Let $\mathcal{B L}_{2, \infty}(\delta, c)$ denote the set of functions in the ball $\mathcal{B L}_{2}(\delta)$ whose integral function belongs to the $c$-ball of $\mathcal{L}_{\infty}$, i.e., $\mathcal{B L}_{2, \infty}(\delta, c) \triangleq\left\{x \in \mathcal{B L}_{2}(\delta):\left\|\int_{0}^{t} x(t) d t\right\|_{\infty}<c\right\}$. The $\mathcal{H}_{\infty}-$ norm of a stable scalar transfer function $T(s)$ is denoted by $\|T\|_{\infty}=\sup _{\omega \in \mathbb{R}}|T(j \omega)|$.

\section{BASIC MODELS AND MotiVATION}

Vehicles in a platoon are indexed by $i=0,1,2, \ldots$ Acceleration, speed, and position of vehicle $i$ are denoted by $a_{i}, v_{i}$, and $p_{i}$, respectively. The lead vehicle is indexed by zero, and it shares $a_{0}, v_{0}$, and $p_{0}$ with other vehicles. According to which form is more suitable for our purpose, systems and signals will be characterized either in the Laplace-domain or in the time-domain.
1) Vehicle Models: The longitudinal vehicle dynamics are time-varying, nonlinear systems. Brakes and driving torques are usually controlled by low level nonlinear controllers. The closed-loop system can be well approximated by low order LTI models

$$
\dot{a}_{i}(t)=-\frac{1}{\tau_{i}} a_{i}(t)+\frac{1}{\tau_{i}}\left(u_{i}\left(t-\Delta_{a, i}\right)+d_{i}(t)\right),
$$

where $u_{i}$ denotes acceleration demand to the low level controller, $\tau_{i}$ denotes time constant, $\Delta_{a, i}$ denotes constant actuator delay, and $d_{i}(t)$ denotes disturbance. Model (1) is widely used for platoon level control design and analysis [3], [8], [20], [21]. In this paper we set $d_{i}(t)=0$. The general case will be analyzed in future works.

2) Control Architectures: Vehicles equipped with radars/lidars are able to measure the distance and relative speed of the predecessor vehicle. Equipped in addition with $\mathrm{V} 2 \mathrm{~V}$ communication abilities, locally measured acceleration, speed and position information can be shared with the follower vehicles. The goal of the Leader and Predecessor Following $(L P F)$ control scheme,

$$
\begin{aligned}
& u_{i}(t)=K_{a, i}^{i-1}\left(a_{i-1}\left(t-\Delta_{i, i-1}\right)-a_{i}\left(t-\Delta_{i, i-1}\right)\right) \\
& \quad+K_{v, i}^{i-1}\left(v_{i-1}\left(t-\Delta_{i, i-1}\right)-v_{i}\left(t-\Delta_{i, i-1}\right)\right)+K_{p, i}^{i-1} e_{i, i-1}(t) \\
& \quad+K_{a, i}^{0}\left(a_{0}\left(t-\Delta_{i, 0}\right)-a_{i}\left(t-\Delta_{i, 0}\right)\right) \\
& \quad+K_{v, i}^{0}\left(v_{0}\left(t-\Delta_{i, 0}\right)-v_{i}\left(t-\Delta_{i, 0}\right)\right)+K_{p, i}^{0} e_{i, 0}\left(t-\Delta_{i, 0}\right),
\end{aligned}
$$

is to simultaneously follow two trajectories, $p_{i}^{\text {ref, }}(t):=$ $p_{0}(t)-R_{i, 0}(t)$ and $p_{i}^{r e f, i-1}(t):=p_{i-1}(t)-R_{i, i-1}(t)$, where $R_{i, j}(t), j \in\{0, i-1\}$, are the desired distances to the leader and the predecessor, respectively, the corresponding spacing errors are defined by

$$
e_{i, j}(t) \triangleq p_{j}(t)-p_{i}(t)-R_{i, j}(t) .
$$

Function $R_{i, j}($.$) is called spacing policy. Controller pa-$ rameters and $\mathrm{V} 2 \mathrm{~V}$ communication delays are denoted by $K_{a, i}^{j}, K_{v, i}^{j}, K_{p, i}^{j}$ and $\Delta_{i, j}, j \in\{0, i-1\}$. In Predecessor Following $(P F)$ control schemes, such as CACC, the last three terms in (2) are missing.

When applying the LPF strategy in well organized, synchronized platoons it is presumed that $p_{i}^{r e f, 0}(t)$ and $p_{i}^{r e f, i-1}(t)$ are close to each other, and cannot be arbitrary functions [3], [22]. In ad hoc heterogeneous platoons we cannot build upon this assumption.

3) Spacing Policies: One of the most common spacing policies in vehicle following is the constant spacing policy (CSP), where $R_{i, j}(t)=L_{i, j}, j \neq i$, is constant. A combination with $\mathrm{PF}$ architecture (common in ACC technology) leads to string instability in a platoon, i.e., oscillations, which are introduced into a traffic flow by braking and accelerating vehicles, may be amplified in the upstream direction. In contrast, LPF control with CSP, $j \in\{0,1\}$, results in string stability with small inter-vehicle gaps [3].

Constant time-headway spacing policy (CTHSP)

$$
R_{i, j}(t)=L_{i, j}+h_{i, j} v_{i}(t), \quad h_{i, j}>0, \quad j \neq i,
$$

allows for string stability of PF architectures with sufficiently large $h_{i, i-1}$ (typically $\in[0.5,1]$ for passenger cars), but we should pay for that with a considerably long inter-vehicle gap. 
Constants $L_{i, j}$ and $h_{i, j}$ denote demanded space at standstill and time-headway, respectively. In the following frequencydomain analysis we assume $L_{i, j}=0$ without loss in generality.

If some vehicles in a platoon have multiple reference trajectories, as in the case of LPF structures, then the issue of consistency of spacing policies emerges [12], which means in general that the set of equations $\left\{p_{i}^{r e f, j}(t)=\right.$ $p_{j}(t)-R_{i, j}(t) \mid \forall i, j$ where $R_{i, j}$ is defined $\}$ is consistent, and $\left\{p_{i}(t)=p_{i}^{r e f, j}(t) \mid \forall i, j\right.$ where $R_{i, j}$ is defined $\}$ is a solution. If a platoon consists of vehicles with PF and LPF architectures, then consistency of spacing policies means that for each vehicle $i$ having LPF architecture

$$
R_{i, 0}(t)=R_{1,0}(t)+R_{2,1}(t)+\ldots+R_{i, i-1}(t), \quad \forall t
$$

is satisfied [12]. Consistency is a necessary condition for the existence of a consensus where all spacing errors are zero.

4) Closed-Loop Model: The closed-loop model is presented in the frequency-domain. Laplace transform of vehicle dynamics (1) (with $d_{i}()=$.0 ) is

$$
a_{i}(s)=H_{i}(s) u_{i}(s), \quad H_{i}(s) \triangleq \frac{1}{\tau_{i} s+1} e^{-s \Delta_{a, i}} .
$$

Control input (2) with (3) and (4) is transformed to

$$
\begin{aligned}
u_{i}(s)= & k_{i}^{i-1}(s)\left(a_{i-1}(s)-a_{i}(s)\right)-k_{h, i}^{i-1}(s) a_{i}(s) \\
& +k_{i}^{0}(s)\left(a_{0}(s)-a_{i}(s)\right)-k_{h, i}^{0}(s) a_{i}(s),
\end{aligned}
$$

where $k_{h, i}^{i-1}(s) \triangleq \frac{K_{p, i}^{i-1} h_{i, i-1}}{s}$ and $k_{h, i}^{0}(s) \triangleq e^{-s \Delta_{i, 0}} \frac{K_{p, i}^{0} h_{i, 0}}{s}$,

$$
\begin{aligned}
k_{i}^{i-1}(s) & \triangleq \frac{1}{s^{2}}\left(s^{2} K_{a, i}^{i-1} e^{-s \Delta_{i, i-1}}+K_{v, i}^{i-1} s+K_{p, i}^{i-1}\right), \\
k_{i}^{0}(s) & \triangleq \frac{1}{s^{2}} e^{-s \Delta_{i, 0}}\left(s^{2} K_{a, i}^{0}+K_{v, i}^{0} s+K_{p, i}^{0}\right) .
\end{aligned}
$$

Inserting (7) into (6), and introducing the following notation

$$
\begin{aligned}
\mathcal{A}_{i}(s) & =H_{i}(s) \mathcal{C}_{u, i}(s), \\
\mathcal{B}_{i}(s) & =H_{i}(s) \mathcal{D}_{u, i}(s), \\
\mathcal{C}_{u, i}(s) & =\frac{k_{i}^{i-1}(s)}{1+H_{i}(s)\left(k_{i}^{i-1}(s)+k_{h, i}^{i-1}(s)+k_{i}^{0}(s)+k_{h, i}^{0}(s)\right)}, \\
\mathcal{D}_{u, i}(s) & =\frac{k_{i}^{0}(s)}{1+H_{i}(s)\left(k_{i}^{i-1}(s)+k_{h, i}^{i-1}(s)+k_{i}^{0}(s)+k_{h, i}^{0}(s)\right)}, \\
\mathcal{C}_{e, i}(s) & =\frac{1}{s^{2}}\left(1-\left(1+s h_{i, i-1}\right) \mathcal{A}_{i}(s)\right), \\
\mathcal{D}_{e, i}(s) & =-\frac{1+s h_{i, i-1}}{s^{2}} \mathcal{B}_{i}(s),
\end{aligned}
$$

yields a general form for the description of components with LPF control architecture:

$$
\begin{aligned}
a_{i}(s) & =\mathcal{A}_{i}(s) a_{i-1}(s)+\mathcal{B}_{i}(s) a_{0}(s), \\
u_{i}(s) & =\mathcal{C}_{u, i}(s) a_{i-1}(s)+\mathcal{D}_{u, i}(s) a_{0}(s), \\
e_{i, i-1}(s) & =\mathcal{C}_{e, i}(s) a_{i-1}(s)+\mathcal{D}_{e, i}(s) a_{0}(s) .
\end{aligned}
$$

5) Heterogeneous Platoons: In this paper, a multi-brand, heterogeneous platoon consists of components described by the general LPF form (16)-(18), where $\mathcal{A}_{i}, \mathcal{B}_{i}, \mathcal{C}_{u, i}, \mathcal{D}_{u, i}, \mathcal{C}_{e, i}$ and $\mathcal{D}_{e, i}$ are arbitrary SISO transfer functions, all but $\mathcal{C}_{e, i}$ and $\mathcal{D}_{e, i}$ are required to be stable. Vehicles with PF control architecture are described with $\mathcal{B}_{i}(s)=\mathcal{D}_{u, i}(s)=\mathcal{D}_{e, i}(s)=0$.
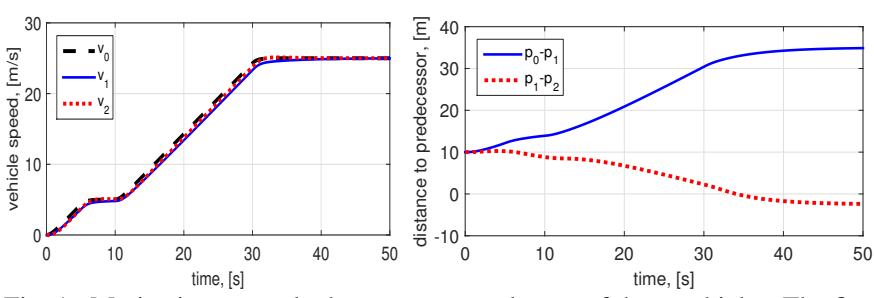

Fig. 1. Motivation example: heterogeneous platoon of three vehicles. The first follower keeps a speed dependent spacing from the leader (PF-CTHSP), the second follower is designed to keep constant distances from both the leader and its predecessor (LPF-CSP).

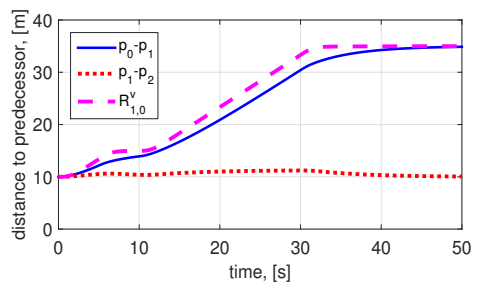

Fig. 2. A solution: the second follower uses adaptive spacing policy (LPFASP). Dashed line denotes the virtual spacing policy of vehicle 1 with respect to the leader. It is computed on the board of vehicle 2 .

The dependence of the transfer functions on the vehicle index indicates the heterogeneity of the platoon: the components may differ in vehicle dynamics, controller, and even spacing policy. In the analysis in Section IV, the specific structure of the transfer functions (10)-(15) are not exploited; therefore, string stability and performance results hold for general LTI vehicle models and controllers.

6) Motivation Example: Spacing problems caused by inconsistent spacing policies are illustrated in this subsection. Given are three vehicles composing a short multi-vendor platoon. The first follower is a CACC vehicle designed to meet string stability requirements. With notations of this paper, it has a PF-CTHSP control architecture $\left(k_{i}^{0}(s)=k_{h, i}^{0}(s)=0\right)$. The second follower vehicle is equipped with an LPF-CSP controller $\left(h_{i, i-1}=h_{i, 0}=0\right)$. It is designed to be string stable in a synchronized platoon, and such that $e_{2,0}(t)+e_{2,1}(t)$ is driven to zero in steady state. The leader with $\tau_{0}=0.7 \mathrm{~s}$ is driven by acceleration demand

$$
u_{0}(t)= \begin{cases}1 & \text { if } t \in[0,5 s] \text { or } t \in[10 s, 30 s] \\ 0 & \text { otherwise. }\end{cases}
$$

The vehicles start from standstill, placed with gaps $L_{1,0}=$ $L_{2,1}=10 \mathrm{~m}$ one after another. The choice $L_{2,0}=20 \mathrm{~m}$ ensures that the spacing errors are initially zero. Fig. 1 illustrates the conflict: follower 1 keeps a speed dependent spacing with time-headway $h_{1,0}=1 s$, while follower 2 is designed to keep constant gaps minimizing both of its spacing errors, $e_{2,0}(t)=$ $p_{0}(t)-p_{2}(t)-L_{2,0}$ and $e_{2,1}(t)=p_{1}(t)-p_{2}(t)-L_{2,1}$, consequently, follower 2 overtakes follower 1 at $t=35 \mathrm{~s}$.

The problem could be resolved if vehicles agreed in the spacing policy. This would require all cars to have a standardized protocol for sharing spacing policies at every joining / leaving maneuver - imposing extra load on the communication network. Then follower 2 would chose $R_{2,0}(t)=L_{2,1}+$ $L_{1,0}+h_{1,0} v_{1}(t)$ (or practically with $v_{1}(t)$ replaced by $v_{2}(t)$ ). Note that there are more specific nonlinear, time-varying, or fault tolerant spacing policies [23], [24], which would be more complicated (if not impossible) to share with the others. 
An alternative self-adjusting and flexible solution is proposed instead: based on measurements already available for control, the joining LPF vehicle estimates the aggregated spacing policy of the preceding vehicles. Fig. 2 demonstrates that by using the adaptive spacing policy described in Section III, follower 2 can keep a small constant gap to follower 1 . Looking at Fig. 2 a question may emerge: is this solution not equivalent to a PF-CSP controller? - Not necessarily. Depending on the design parameters, ASP allows the utilization of leader information for improving tracking performance and achieving string stability. The answer is discussed more deeply in the following sections.

\section{LEADER AND PREdeCESSOR Following CONTROL With ADAPTIVE SPACING POLICY (LPF-ASP)}

The structure of the proposed LPF-ASP control system is presented in this section. The LPF type vehicle is placed in an arbitrary platoon at position $i$. It is assumed that measurements for $a_{0}, v_{0}, p_{0}, a_{i-1}, v_{i-1}$ and $p_{i-1}$ are available. The goals of the control are to follow the predecessor within small intervehicle gaps and to satisfy conditions for string stability. These goals are achieved by the on-line modification of the spacing policy with respect to the leader according to the behavior of the preceding platoon, and by the appropriate choice of design parameters.

The first goal is to make the spacing policies consistent, so we seek $R_{i, 0}$ in the form $R_{i, 0}(t)=R_{i-1,0}(t)+R_{i, i-1}(t)$, where $R_{i, i-1}(t)$ is our free choice, but $R_{i-1,0}(t)$, the spacing policy of the preceding vehicle with respect to the leader is unknown; moreover, it is undefined in general (for example when human driven vehicles are also mingled in the platoon). Given $R_{i, i-1}(t)$, the spacing policy with respect to the leader is determined by the spacing policy adaptation law

$$
R_{i, 0}(t)=R_{i-1,0}^{v}(t)+R_{i, i-1}(t),
$$

where $R_{i-1,0}^{v}(t)$ is called virtual spacing policy, it reflects the behavior of the preceding platoon and is computed on the board of vehicle $i$ based on the available measurements.

1) Simplest Form for $R_{i-1,0}^{v}(t)$ : The simplest virtual spacing policy of the predecessor could be $R_{i-1,0}^{v}(t) \triangleq p_{0}(t)-$ $p_{i-1}(t)$. It follows by the spacing policy adaptation law that $R_{i, 0}(t)=p_{0}(t)-p_{i-1}(t)+R_{i, i-1}(t)$. This yields that the two reference positions coincide by definition, $p_{i}^{r e f, 0}(t) \triangleq p_{0}(t)-$ $R_{i, 0}(t)=p_{0}(t)-p_{0}(t)+p_{i-1}(t)-R_{i, i-1}(t) \triangleq p_{i}^{r e f, i-1}(t)$, so we lose the freedom of LPF structures, and we actually have a PF control structure, where string stability cannot be achieved with CSP.

In order to achieve string stability, $R_{i-1,0}^{v}(t)$ must be constructed dynamically in the form

$$
R_{i-1,0}^{v}(s)=\left[\begin{array}{ll}
E_{i, 0}(s), & E_{i, i-1}(s)
\end{array}\right]\left[\begin{array}{c}
a_{0}(s) \\
a_{i-1}(s)
\end{array}\right] .
$$

Explicit requirements on the choice of transfer functions $E_{i, 0}$ and $E_{i, i-1}$ are not formulated. The ultimate goals are string stability, small control effort and small spacing errors of the overall LPF-ASP system.

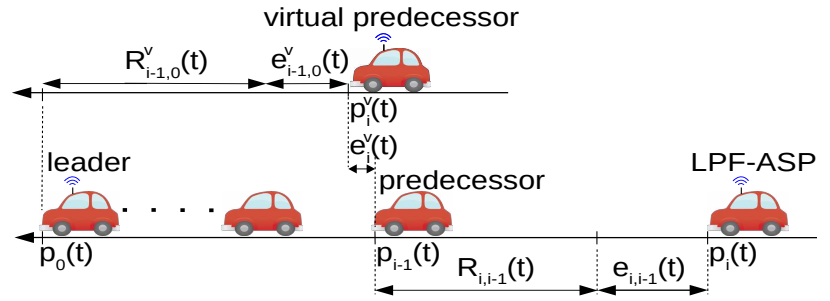

Fig. 3. Concept of virtual predecessor (VP) model. VP is a PF type virtual vehicle that follows the leader according to the spacing policy given by $R_{i-1,0}^{v}$ and moves close to the predecessor vehicle $i-1$.

2) Basic Idea: A possible concept to chose $R_{i-1,0}^{v}$ was introduced in [12]: pick up a virtual predecessor (VP) vehicle model of PF structure that follows directly the leader with a virtual spacing policy $R_{i-1,0}^{v}(t)$, see also Fig. 3 . The VP model is driven by two inputs: 1) the motion of the leader, $a_{0}(t)$, which is given, and 2) its desired spacing policy input, $R_{i-1,0}^{v}(t)$. If we require that the VP model moves very close to vehicle $i-1$, then we have to find an appropriate input $R_{i-1,0}^{v}(t)$ that enforces this motion. Then, from the point of view of vehicle $i$, the unknown policy $R_{i-1,0}(t)$ of the true predecessor can be replaced by the virtual policy $R_{i-1,0}^{v}(t)$ of the VP model.

The designer has some freedom in choosing the VP model and the method for computing an appropriate virtual spacing policy $R_{i-1,0}^{v}(t)$ [12], [17]. Even the accurate, simultaneous motion of VP and the predecessor is not an absolute necessity, see the case described in [17] for an example. The choice of the dynamics of VP and its virtual spacing policy, however, influences the closed-loop dynamics and spacing errors of vehicle $i$. A construction method for $R_{i-1,0}^{v}(t)$ is derived in the following.

3) VP Structure: The proposed structure is simple yet flexible enough to provide insight into the approach while allowing design freedom to achieve sufficient tracking performance and string stability. From (1)-(3) the PF type VP model is described by the following equations

$$
\begin{aligned}
\dot{a}_{i}^{v}(t)= & -\frac{1}{\tau_{i}^{v}} a_{i}^{v}(t)+\frac{1}{\tau_{i}^{v}} u_{i}^{v}(t), \\
u_{i}^{v}(t)= & K_{a, i}^{v}\left(a_{0}(t)-a_{i}^{v}(t)\right)+K_{v, i}^{v}\left(v_{0}(t)-v_{i}^{v}(t)\right) \\
& +K_{p, i}^{v}\left(p_{0}(t)-p_{i}^{v}(t)\right)-K_{p, i}^{v} R_{i-1,0}^{v}(t),
\end{aligned}
$$

where $a_{i}^{v}, v_{i}^{v}, p_{i}^{v}$ and $u_{i}^{v}$ denote respectively acceleration, speed, position and control input of the VP model, $\tau_{i}^{v}, K_{a, i}^{v}, K_{v, i}^{v}$ and $K_{p, i}^{v}$ are positive design parameters. Index $i$ expresses that the VP model belongs to the control system of vehicle $i$. The VP model follows directly the leader with spacing error $e_{i-1,0}^{v}(t) \triangleq p_{0}(t)-p_{i}^{v}(t)-R_{i-1,0}^{v}(t)$. It is driven by two kinds of inputs: 1$)$ the motion variables of the leader and 2) the unknown spacing policy, $R_{i-1,0}^{v}(t)$. The next goal is to find $R_{i-1,0}^{v}(t)$ which makes the VP move close to the true predecessor.

4) Computation of $R_{i-1,0}^{v}(t)$ : When the VP model moves together with the true predecessor thanks to some appropriate input $R_{i-1,0}^{v}(t)$, then the virtual motion variables $a_{i}^{v}, v_{i}^{v}$ and $p_{i}^{v}$ in (23) are equal to the respective variables $a_{i-1}, v_{i-1}$ and 


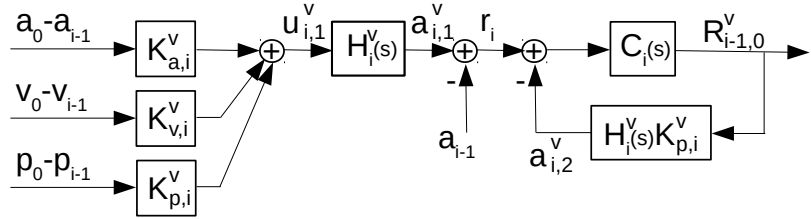

Fig. 4. Construction of virtual spacing policy $R_{i-1,0}^{v}$ that minimizes the virtual tracking error $e_{i}^{v}=p_{i}^{v}-p_{i-1}$.

$p_{i-1}$. In this case the virtual control law (23) can be written as $u_{i}^{v}(t)=u_{i, 1}^{v}(t)+u_{i, 2}^{v}(t)$, where

$$
\begin{aligned}
u_{i, 1}^{v}(t)= & K_{a, i}^{v}\left(a_{0}(t)-a_{i-1}(t)\right)+K_{v, i}^{v}\left(v_{0}(t)-v_{i-1}(t)\right) \\
& +K_{p, i}^{v}\left(p_{0}(t)-p_{i-1}(t)\right)
\end{aligned}
$$

is known from measurements, but

$$
u_{i, 2}^{v}(t)=-K_{p, i}^{v} R_{i-1,0}^{v}(t)
$$

is yet to be determined. VP model (22)-(25) can be formulated as the superposition of the following two systems,

$$
\begin{array}{ll}
\Sigma_{1}: & \dot{a}_{i, 1}^{v}(t)=-\frac{1}{\tau_{i}^{v}} a_{i, 1}^{v}(t)+\frac{1}{\tau_{i}^{v}} u_{i, 1}^{v}(t), \\
\Sigma_{2}: & \dot{a}_{i, 2}^{v}(t)=-\frac{1}{\tau_{i}^{v}} a_{i, 2}^{v}(t)+\frac{K_{p, i}^{v}}{\tau_{i}^{v}} R_{i-1,0}^{v}(t) .
\end{array}
$$

For an appropriate input $R_{i-1,0}^{v}$ we have $a_{i}^{v}(t)=a_{i-1}(t)$ after some transient time $t>t_{1}$, which is due to the different initial conditions $a_{i}^{v}(0)$ and $a_{i-1}(0)$. Thus, by superposition

$$
a_{i}^{v}(t)=a_{i, 1}^{v}(t)-a_{i, 2}^{v}(t)=a_{i-1}(t)
$$

hold for $t>t_{1}$. The right equality of (28) defines a reference signal

$$
r_{i}(t) \triangleq a_{i, 1}^{v}(t)-a_{i-1}(t)
$$

for the output, $a_{i, 2}^{v}(t)$, of system $\Sigma_{2}$. We look for $R_{i-1,0}^{v}$ that minimizes $\left|r_{i}(t)-a_{i, 2}^{v}(t)\right|$. In this way the spacing policy construction problem is transformed into a reference tracking control problem where $\Sigma_{2}$ is the plant, equations

$$
\begin{aligned}
\ddot{e}_{i, 2}^{v}(t) & \triangleq r_{i}(t)-a_{i, 2}^{v}(t), \\
R_{i-1,0}^{v}(t) & =C_{a, i} \ddot{e}_{i, 2}^{v}(t)+C_{v, i} \dot{e}_{i, 2}^{v}(t)+C_{p, i} e_{i, 2}^{v}(t),
\end{aligned}
$$

define a possible feedback controller, $R_{i-1,0}^{v}(t)$ is produced as the control signal and $C_{a, i}, C_{v, i}, C_{p, i}>0$ are controller coefficients. Initial condition for $a_{i, 2}^{v}$ and $e_{i, 2}^{v}$ are set such that $R_{i-1,0}^{v}(0)=p_{0}(0)-p_{i-1}(0)$. This control scheme minimizes the discrepancy, $e_{i}^{v}(t) \triangleq p_{i}^{v}(t)-p_{i-1}(t)$, between the motion of the virtual and the true predecessor. The block scheme is presented in Fig. 4, where the transfer functions are defined by $H_{i}^{v}(s) \triangleq \frac{1}{\tau_{v}^{v} s+1}$ and $C_{i}(s) \triangleq \frac{C_{a, i} s^{2}+C_{v, i} s+C_{p, i}}{s^{2}}$. It is emphasized in Fig. 4 that the required information is $a_{0}, a_{i-1}$, $v_{0}-v_{i-1}$ and $p_{0}-p_{i-1}$, which is already available also in classical LPF control schemes.

5) Closed-Loop Model: Spacing policy $R_{i, i-1}(t)$ for LPFASP vehicles is chosen to be constant in this paper. It plays similar role in the analysis as initial vehicle positions and so it is omitted. The above derivation yields the following transfer functions for the closed-loop LPF-ASP model, $\mathcal{A}_{i}(s)=H_{i}(s) \mathcal{C}_{u, i}(s), \mathcal{B}_{i}(s)=H_{i}(s) \mathcal{D}_{u, i}(s)$, and

$$
\begin{aligned}
\mathcal{C}_{u, i}(s) & =\frac{k_{i}^{i-1}(s)-K_{p, i}^{0} E_{i, i-1}(s)}{1+H_{i}(s)\left(k_{i}^{0}(s)+k_{i}^{i-1}(s)\right)}, \\
\mathcal{D}_{u, i}(s) & =\frac{k_{i}^{0}(s)-K_{p, i}^{0} E_{i, 0}(s)}{1+H_{i}(s)\left(k_{i}^{0}(s)+k_{i}^{i-1}(s)\right)}, \\
\mathcal{C}_{e, i}(s) & =\frac{1}{s^{2}}\left(1-\mathcal{A}_{i}(s)\right), \\
\mathcal{D}_{e, i}(s) & =-\frac{1}{s^{2}} \mathcal{B}_{i}(s),
\end{aligned}
$$

where $k_{i}^{i-1}$ and $k_{i}^{0}$ are defined respectively by

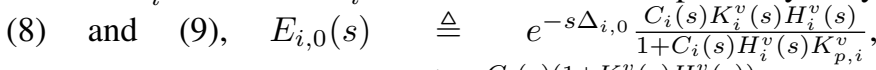
$E_{i, i-1}(s) \triangleq \quad-e^{-s \Delta_{i, 0}} \frac{C_{i}(s)\left(1+K_{i}^{v}(s) H_{i}^{v}(s)\right)}{1+C_{i}(s) H_{i}^{v}(s) K_{p, i}^{v}} \quad$ with $K_{i}^{v}(s) \triangleq \frac{K_{a, i}^{v} s^{2}+K_{v, i}^{v} s+K_{p, i}^{v}}{s^{2}}$. It is taken into consideration in $E_{i, 0}(s)$ and $E_{i, i-1}(s)$ that the computed virtual spacing policy depends on delayed information. Stability of $\mathcal{C}_{u, i}$ and $\mathcal{D}_{u, i}$ require that both $s^{3} \tau_{i}^{v}+s^{2}\left(1+C_{a, i} K_{p, i}^{v}\right)+s C_{v, i} K_{p, i}^{v}+C_{p, i} K_{p, i}^{v}$ and $s^{3} \tau_{i}+s^{2}\left(1+K_{a, i}^{0}+K_{a, i}^{i-1}\right)+s\left(K_{v, i}^{0}+K_{v, i}^{i-1}\right)+K_{p, i}^{0}+K_{p, i}^{i-1}$ be stable polynomials. By the Routh-Hurwitz stability criterion [25, Section III.8], the parameters must satisfy $\left(1+C_{a, i} K_{p, i}^{v}\right) C_{v, i}>\tau_{i}^{v} C_{p, i}$ and $\left(1+K_{a, i}^{0}+K_{a, i}^{i-1}\right)\left(K_{v, i}^{0}+K_{v, i}^{i-1}\right)>\tau_{i}\left(K_{p, i}^{0}+K_{p, i}^{i-1}\right)$.

\section{StRing Stability}

The goal of this section is to analyze the string stability of the heterogeneous platoon of vehicles (16)-(18) and, based on the analysis, derive constraints for the design of LPF-ASP controllers. It will be shown with the help of a recursive description of vehicle strings that the notion of string stability leads to a distributed condition, i.e., each member of the platoon has to satisfy a local condition without the need for respecting dynamics of other vehicles.

Since LPF-ASP vehicles are intended to work in general heterogeneous, ad hoc platoons, and the related theoretical results are not fully elaborated in the literature, some new definitions and theorems need be introduced in order to evaluate the performance of LPF-ASP controllers. One of the main directions for proving string stability is the (vector-) Lyapunov function based approach, which is powerful in the analysis of general heterogeneous platoons in terms of nonzero initial conditions [26], but the effects of inputs and synthesis conditions have not been elaborated within this framework.

In contrast, the transfer function / performance oriented approaches are useful in analyzing the effects of reference signals and disturbances, but have some limitations when facing with heterogeneity. Within this framework, the existence of local string stability conditions can be derived for some particular structures, if a so called spacing error transfer function, $\Gamma_{i}(s)$, can be defined between the subsequent spacing errors, $e_{i, i-1}(s) \triangleq \Gamma_{i}(s) e_{i-1, i-2}(s)$, and this transfer function depends only on the parameters of vehicle $i$ [9], [11]. A sufficient condition for string stability is then $\left\|\Gamma_{i}\right\|_{\infty} \leq 1$. Unfortunately, there is no such transfer function in general, see references [8], [20], where $\Gamma_{i}(s)$, as defined above, depends on all vehicles $0,1,2, \ldots, i$. There are three further problems with this approach as can be seen in the light of the results in this section: 1) $\Gamma_{i}$ is not necessarily stable, yet the platoon 
might work well; 2) in a heterogeneous platoon of general vehicle structures $\left\|\Gamma_{i}\right\|_{\infty} \leq 1$ should be tested robustly for all possible permutations of the vehicle ordering; 3) in general, $\left\|\Gamma_{i}\right\|_{\infty} \leq 1$ does not lead to synthesis conditions.

\section{A. Recursive Description of Vehicle Strings}

When analyzing string stability we are usually interested in the spatial evolution of performance variables such as spacing errors, accelerations and control effort. Classical performance oriented approaches try to give direct relations between the consecutive performance variables, for instance in the form $e_{i, i-1}(s)=\Gamma_{i}(s) e_{i-1, i-2}(s)$. In contrast, there are two kinds of variables in the method of recursive description: 1) variables transmitted from vehicle to vehicle, and 2) performance variables of interest.

Examples for the first group are vehicle acceleration (in this paper), or control effort (in [9]). (Though vehicle speed and position are physically transmitted variables too, they can be formally computed by integration at the model of the receiver vehicle; therefore, they do not appear explicitly in the recursion model.) Transmitted variables play the role of spacedomain state-variables in the recursion model. Examples for the performance variables are spacing errors and control effort. They play the role of output variables in the recursion model.

Consider the vehicle models in the form (16)-(18) and introduce the following first to end transfer functions

$$
\begin{aligned}
a_{i}(s) & =\mathcal{G}_{i}(s) a_{0}(s), \\
u_{i}(s) & =\mathcal{F}_{u, i}(s) a_{0}(s), \\
e_{i, i-1}(s) & =\mathcal{F}_{e, i}(s) a_{0}(s),
\end{aligned}
$$

which, starting from $\mathcal{G}_{0}(s)=1$, evolve with vehicle index as

$$
\left[\begin{array}{c}
\mathcal{G}_{i}(s) \\
\hline \mathcal{F}_{u, i}(s) \\
\mathcal{F}_{e, i}(s)
\end{array}\right]=\left[\begin{array}{c|c}
\mathcal{A}_{i}(s) & \mathcal{B}_{i}(s) \\
\hline \mathcal{C}_{u, i}(s) & \mathcal{D}_{u, i}(s) \\
\mathcal{C}_{e, i}(s) & \mathcal{D}_{e, i}(s)
\end{array}\right]\left[\begin{array}{c}
\mathcal{G}_{i-1}(s) \\
\hline 1
\end{array}\right],
$$

$i=1,2,3, \ldots$. The complex difference equation (39) is the recursive description of the heterogeneous platoon, since multiplying both sides by $a_{0}(s)$, the evolution of $a_{i}(s), u_{i}(s)$ and $e_{i, i-1}(s), i=1,2,3, \ldots$, can be computed recursively.

\section{B. Definitions for String Stability}

The transient behavior of the platoon is examined as the effect of leader maneuver $a_{0}(t)$ that is assumed to belong to one of the following admissible sets.

Definition 1 (Admissible Leader Maneuvers):

1) Bounded energy acceleration: $a_{0} \in \mathcal{B L}_{2}(\delta)$

2) Bounded energy acceleration and limited speed: $a_{0} \in$ $\mathcal{B L}_{2, \infty}(\delta, c)$.

In case of ad hoc, unorganized platoons we have to be prepared for the worst case of vehicle ordering, so a robust version of the classical string stability definitions must be considered. In the following definition, which is an adaptation of [21, Definition 1], $a_{i}$ can be replaced by any variable of interest, such as $u_{i}$ or $e_{i, i-1}$.

Definition 2: Vehicle platoon (39) is heterogeneous (or robustly) string stable with respect to $a_{i}$ in the $\mathcal{L}_{2}$ norm, if for each $\delta>0$ there exist a finite scalar $L(\delta)$ such that $\left\|a_{i}\right\|_{2}<L$ is satisfied for all $i>0$, for any bounded leader maneuvers, $a_{0} \in \mathcal{B} \mathcal{L}_{2}(\delta)$, and for arbitrary ordering of the vehicles.

Remark 1: String stability in the strict sense, as defined in [3], [20], i.e., $\left\|e_{i, i-1}\right\|_{2}<\left\|e_{i-1, i-2}\right\|_{2}$ for all $i>0$, cannot be expected in general due to the link with the leader and by heterogeneity of the platoon, see [21] and [27] for counterexamples, and see also Remark 4 . In contrast to $\mathcal{L}_{p^{-}}$ string stability defined in [20], Definition 2 is independent on the system that generates the signals and it requires the boundedness of signals for all vehicle ordering.

It will be shown that boundedness of spacing errors in the $\mathcal{L}_{2}$ norm for arbitrary $\mathcal{L}_{2}$ input is too strict requirement in some (pathological) cases presented in Section IV-E, where the spacing error is written as the sum of two terms, one is bounded in the $\mathcal{L}_{\infty}$ norm and the other is bounded in the $\mathcal{L}_{2}$ norm. In those cases the following milder notion for string stability can be proved provided that the leader maneuver is restricted to the more practical set, $\mathcal{B L}_{2, \infty}(\delta, c)$.

Definition 3: Vehicle platoon (39) is ultimately heterogeneous string stable with respect to $e_{i, i-1}$, if for all $\delta>0$ and $c>0$ there exist scalars $T(\delta, i)$ and $L(\delta, c)$ such that for arbitrary bounded leader maneuvers, $a_{0} \in \mathcal{B} \mathcal{L}_{2, \infty}(\delta, c)$, and for arbitrary ordering of the vehicles, the spacing errors $e_{i, i-1}$ remain uniformly bounded by $L(\delta, c)$ after time $t>T(\delta, i)$, i.e. $\max _{t>T(\delta, i)}\left|e_{i, i-1}(t)\right|<L(\delta, c)$, for all $i>0$.

\section{General Conditions for String Stability}

For any fixed complex variable $s$, (39) defines a spatially discrete and varying linear dynamical system over the complex field, driven by constant input 1 , and with initial condition $\mathcal{G}_{0}(s)=1$. This implies that string stability of the platoon is related to the stability of discrete (in the spatial index $i$ ) linear systems where the variation of the coefficients is due to the heterogeneity of the platoon. The following theorem is a straightforward adaptation of [28, Theorem 1].

Theorem 1: The vehicle platoon (39) is heterogeneous string stable with respect to $a_{i}$ in the $\mathcal{L}_{2}$ norm (Definition 2) if and only if all of the following conditions hold for all $i>0$ :

1) $\left|\mathcal{A}_{i}(j \omega)\right| \leq 1$ for all $\omega$, where $\mathcal{B}_{i}(j \omega)=0$,

2) $\left|\mathcal{A}_{i}(j \omega)\right|<1$ for all $\omega$, where $\left|\mathcal{B}_{i}(j \omega)\right|>0$,

3) $\left|\mathcal{B}_{i}(j \omega)\right|$ is finite for all $\omega$.

Proof. Uniform boundedness of $a_{i}$ in the $\mathcal{L}_{2}$ norm is equivalent to the uniform boundedness of transfer functions $\mathcal{G}_{i}$ in the $\mathcal{H}_{\infty}$ norm. Technical details can be found in [28]. The proof is based on the fact that for every $\omega$ the solution of the one-dimensional linear system with bounded spatiallyvarying uncertainty and bounded input is bounded if and only if the system is robustly stable with respect to the spatial variations. If $\mathcal{A}_{i} \mathcal{A}_{j}=\mathcal{A}_{j} \mathcal{A}_{i}$ for all $i$ and $j$, and now this is the case since $\mathcal{A}_{i}$ is scalar, then robust stability of a system with varying coefficients is equivalent to the stability of every spatially invariant system $\mathcal{G}_{i}(s)=\mathcal{A}_{k}(s) \mathcal{G}_{i-1}(s), \forall k$ fixed [29].

Since the spatial state variable $a_{i}(s)$ is scalar, it follows that the string stability conditions for homogeneous and heterogeneous platoons coincide. An important consequence is that the 
string stability constraints imposed on the control design are independent for every vehicles, and this is true even for general components (16)-(18).

Concerning the performance outputs, $u_{i}$, the following condition is sufficient in practical systems to establish their uniform boundedness in the $\mathcal{L}_{2}$ norm, or equivalently, of transfer functions $\mathcal{F}_{u, i}$ in the $\mathcal{H}_{\infty}$ norm.

Theorem 2: The vehicle platoon (39) is heterogeneous string stable with respect to $u_{i}$ in the $\mathcal{L}_{2}$ norm (Definition 2) if all of the following conditions hold

1) the vehicle platoon (39) is heterogeneous string stable with respect to $a_{i}$ in the $\mathcal{L}_{2}$ norm (Definition 2),

2) both $\left|\mathcal{C}_{u, i}(j \omega)\right|$ and $\left|\mathcal{D}_{u, i}(j \omega)\right|$ are bounded for all $\omega$.

Proof. $\left\|\mathcal{F}_{u, i}\right\|_{\infty} \leq \max _{i}\left\{\left\|\mathcal{C}_{u, i}\right\|_{\infty}\left\|\mathcal{G}_{i-1}\right\|_{\infty}+\left\|\mathcal{D}_{u, i}\right\|_{\infty}\right\}$.

\section{Platoon Dependent Conditions for String Stability}

Theorem 2 cannot be adapted to analyze string stability with respect to spacing errors in case of LPF-ASP vehicles, because $\mathcal{C}_{e, i}$, and $\mathcal{D}_{e, i}$ contain integrators, see Fig. 5 . It will be shown that the uniform boundedness properties of the spacing errors depend on the quality of the preceding platoon. This "quality" is characterized by the following notion.

Definition 4 (Platoon Classification): A vehicle platoon of length $n$ (not counting the leader) is said to be of type $k$ if the number of zeros of transfer function $1-\mathcal{G}_{n}(s)$ at $s=0$ is $k$.

Remark 2: The notion is analogous to the notion type $k$ of feedback systems, where $k$ is the number of integrators of the open-loop plant that is feed back through a unit gain [25]. With reference input $a_{0}$ and output $a_{n}$ the sensitivity function is $1-\mathcal{G}_{n}$, while the open-loop plant is $\frac{\mathcal{G}_{n}}{1-\mathcal{G}_{n}}$. This shows that the zeros of $1-\mathcal{G}_{n}$ at $s=0$ are the integrators of the open-loop plant. Having one integrator in the open-loop ensures accurate steady state tracking of unit step reference $a_{0}$ or equivalently accurate steady state tracking of speed, $v_{0}$, for impulsive $a_{0}$. Having two integrators in the open-loop ensures accurate tracking in the position, $p_{0}$, for impulsive $a_{0}$. In short, the system mapping $a_{0}$ to $p_{0}-p_{n}$ is stable for platoons of type 2 , and contains one integrator for platoons of type 1 . It will be clear from Lemma 2 that the notion type $k$ is related to the notion of consensus between the components, i.e., $v_{i} \rightarrow v_{0}$ for $k=1$ and $\left(v_{i} \rightarrow v_{0}, p_{i} \rightarrow p_{0}\right)$ for $k=2$, if $a_{0} \rightarrow 0$.

Lemma 1: A platoon is of type $k$ with $k>0$ if and only if $\mathcal{G}_{n}(j 0)=1$.

The proof is trivial. In order to ease the classification of platoons based on the types of vehicles, Definition 4 is adapted to single vehicles. The corresponding feedback system for which the analogy mentioned in Remark 2 exists is obtained by making the two references $a_{i-1}$ and $a_{0}$ equal.

Definition 5 (Vehicle Classification): Vehicle (16)-(18) is said to be of type $k$ if $k$ is the number of zeros of transfer function $1-\mathcal{A}_{i}(s)-\mathcal{B}_{i}(s)$ at $s=0$.

Remark 3: It is easy to see that PF-CSP, LPF-CSP and LPFASP (with $R_{i, i-1}(t)$ constant) vehicles are of type 2, while PF-CTHSP vehicles are of type 1 .

It is shown in the following how type $k$ of the platoon evolves when new vehicles join the platoon.
Lemma 2 (Type Evolution): Assume that $\mathcal{A}_{i}(s)$ has no zeros at $s=0$. Let vehicle $i$ of type $k_{i}$ join a platoon of length $i-1$ that is of type $K_{i-1}$. Then the resulted platoon of length $i$ is of type $K_{i}=\min \left\{K_{i-1}, k_{i}\right\}$.

The assumption that $\mathcal{A}_{i}$ has no zeros at zero is naturally satisfied by all practical vehicle following algorithms, and simplifies the statement.

Proof. The type of the platoon of length $i$ is defined by the number of zeros of $1-\mathcal{G}_{i}$ at $s=0$, but $1-\mathcal{G}_{i}(s)=$ $1-\left(\mathcal{A}_{i}(s) \mathcal{G}_{i-1}(s)+\mathcal{B}_{i}(s)\right)$, where for $\mathcal{G}_{i-1}$ it is true that there exists a stable transfer function $W_{i-1}$ having no zeros at $s=0$ such that $1-\mathcal{G}_{i-1}(s)=s^{K_{i-1}} W_{i-1}(s)$. It follows that $1-\mathcal{G}_{i}(s)=1-\mathcal{A}_{i}(s)-\mathcal{B}_{i}(s)+s^{K_{i-1}} \mathcal{A}_{i}(s) W_{i-1}(s)$. Finally, from the assumption that vehicle $i$ is of type $k_{i}$ it follows that there exists a stable transfer function $V_{i}$ having no zeros at $s=0$ such that $1-\mathcal{A}_{i}(s)-\mathcal{B}_{i}(s)=s^{k_{1}} V_{i}(s)$, thus $1-\mathcal{G}_{i}(s)=s^{K_{i}} W_{i}(s)$, where $W_{i}(s):=s^{k_{i}-K_{i}} V_{i}(s)+$ $s^{K_{i-1}-K_{i}} \mathcal{A}_{i}(s) W_{i-1}(s)$ is stable and has no zeros at zero. $\square$

The lemma can be interpreted as follows. The tracking property of the platoon is determined by the vehicle of the weakest tracking property. The following theorem shows that whenever the platoon is string stable with respect to the transmitted variable $\left(a_{i}\right)$, then the boundedness of spacing error $e_{i, i-1}$ of the particular vehicle $i$ depends only on the properties of vehicle $i$ and the type of the preceding platoon.

Theorem 3: Suppose that platoon (39) is heterogeneous string stable with respect to $a_{i}$ in the sense of Definition 2 . Suppose that the platoon is of type $K \geq 0$.

1) Assume that

a) $\mathcal{C}_{e, i}(s)$ has at most $K$ integrators, but the other poles are stable,

b) transfer function $\mathcal{C}_{e, i}(s)+\mathcal{D}_{e, i}(s)$ is stable.

Then $\mathcal{F}_{e, i} \in \mathcal{H}_{\infty}$.

2) If condition of statement 1) is satisfied for all vehicles, then the bound for $\mathcal{F}_{e, i}$ is uniform, i.e., the platoon is heterogeneous string stable with respect to spacing error $e_{i, i-1}$ in the sense of Definition 2 .

Proof. The boundedness and string stability of spacing errors is related to the properties of $\mathcal{F}_{e, i}(s)=\mathcal{C}_{e, i}(s) \mathcal{G}_{i-1}(s)+$ $\mathcal{D}_{e, i}(s)$. By string stability with respect to $a_{i}$, sequence $\mathcal{G}_{i}(s)$, $i \geq 0$, is uniformly bounded in the $\mathcal{H}_{\infty}$ norm. By the type condition of the platoon, there exist transfer functions $W_{i-1}(s), i>0$, uniformly bounded in the $\mathcal{H}_{\infty}$ norm such that for all $i$ we have $\mathcal{G}_{i-1}(s)=1-s^{K} W_{i-1}(s)$. It follows that $\mathcal{F}_{e, i}(s)=\mathcal{C}_{e, i}(s)+\mathcal{D}_{e, i}(s)-s^{K} \mathcal{C}_{e, i}(s) W_{i-1}(s)$, where both $\mathcal{C}_{e, i}(s)+\mathcal{D}_{e, i}(s)$ and $s^{K} \mathcal{C}_{e, i}(s) W_{i-1}(s)$ are bounded in the $\mathcal{H}_{\infty}$ norm, which proves 1$)$. The second statement follows trivially.

PF-CSP, PF-CTHSP, LPF-CSP and LPF-ASP vehicles all satisfy that $\mathcal{C}_{e, i}(s)+\mathcal{D}_{e, i}(s)$ is stable. For the LPF-ASP architecture, however, both $\mathcal{C}_{e, i}(s)$ and $\mathcal{D}_{e, i}(s)$ have one integrator, which implies that an LPF-ASP vehicle has bounded $\mathcal{L}_{2}$ spacing error only if the preceding platoon is of type $k>0$.

\section{E. String Stability with LPF-ASP Vehicles}

In this section LPF-ASP vehicles are evaluated in situations where preceding vehicles does not necessarily follow each 
other: 1) In type 0 platoons the vehicles may travel at different steady-state speed (low density traffic with human driven vehicles); 2) the platoon is broken up unexpectedly.

If the platoon is of type $0, \mathcal{G}_{i-1}(j 0) \neq 1$ by Lemma 1 . Let $g_{i-1}$ denote the constant $G_{i-1}(j 0)-1$ and write the preceding platoon dynamics in the form $\mathcal{G}_{i-1}(s)=\left(\mathcal{G}_{i-1}(s)-g_{i-1}\right)+$ $g_{i-1}$, where the first term is a platoon of type 1 . If vehicle $i$ is an LPF-ASP vehicle, then its spacing error reveals the following form

$$
\begin{aligned}
& e_{i, i-1}(s)=e_{i, i-1}^{\left(\mathcal{L}_{2}\right)}(s)+e_{i, i-1}^{\left(\mathcal{L}_{\infty}\right)}(s), \\
& e_{i, i-1}^{\left(\mathcal{L}_{2}\right)}(s)=\left(\mathcal{C}_{e, i}(s)\left(\mathcal{G}_{i-1}(s)-g_{i-1}\right)+\mathcal{D}_{e, i}(s)\right) a_{0}(s), \\
& e_{i, i-1}^{\left(\mathcal{L}_{\infty}\right)}(s)=\mathcal{C}_{e, i}(s) g_{i-1} a_{0}(s)
\end{aligned}
$$

where $e_{i, i-1}^{\left(\mathcal{L}_{2}\right)} \in \mathcal{L}_{2}$ based on Theorem 3. Since $\mathcal{C}_{e, i}$ contains one integrator, $\tilde{\mathcal{C}}_{e, i}(s) \triangleq s \mathcal{C}_{e, i}(s)$ is a stable finite dimensional transfer function. It follows that $e_{i, i-1}^{\left(\mathcal{L}_{\infty}\right)}(s)=\tilde{\mathcal{C}}_{e, i}(s) g_{i-1} v_{0}(s)$, which further implies that $e_{i, i-1}^{\left(\mathcal{L}_{\infty}\right)} \in \mathcal{L}_{\infty}$ if $v_{0} \in \mathcal{L}_{\infty}$.

Theorem 4: Suppose that platoon (39) is heterogeneous string stable with respect to $a_{i}$ in the sense of Definition 2 . Suppose that the platoon is of type 0. Assume that for each vehicle $\mathcal{C}_{e, i}(s)$ has at most 1 integrator, but the other poles are stable, and transfer function $\mathcal{C}_{e, i}(s)+\mathcal{D}_{e, i}(s)$ is stable. Then the platoon is ultimately heterogeneous string stable with respect to spacing error $e_{i, i-1}$ in the sense of Definition 3 .

Proof. We have already shown that the spacing error can be written as the sum of an $\mathcal{L}_{2}$ and an $\mathcal{L}_{\infty}$ signal. It remained to show that there exist constants $T(\delta, i)$ and $L(\delta, c)$ such that $\max _{t>T(\delta, i)}\left|e_{i, i-1}(t)\right|<L(\delta, c)$, whenever $a_{0} \in \mathcal{B} \mathcal{L}_{2, \infty}(\delta, c)$. Since $e_{i, i-1}^{\left(\mathcal{L}_{2}\right)} \in \mathcal{L}_{2}$, there exists a constant $T(\delta, i)$ for every $L_{1}>0$ such that $\left|e_{i, i-1}^{\left(\mathcal{L}_{2}\right)}(t)\right|<L_{1}$ for all $t>T(\delta, i)$. Then $L(\delta, c):=L_{1}+\left\|e_{i, i-1}^{\left(\mathcal{L}_{\infty}\right)}\right\|_{\infty}$.

Remark 4: Theorems 3 and 4 state that string stability with respect to the acceleration is a necessary condition for string stability with respect to the spacing errors. The main character (boundedness in $\mathcal{L}_{2}$, or $\mathcal{L}_{\infty}$ or divergence) of the spacing error of vehicle $i$ depend on the properties of vehicle $i$. It is possible, for instance, that $e_{i, i-1} \in \mathcal{L}_{\infty}$ while $e_{i+1, i} \in \mathcal{L}_{2}$ and yet the platoon might work well. This is one argument against testing the spacing error transfer function, $\Gamma_{i}(s):=\frac{e_{i-1, i-2}(s)}{e_{i, i-1}(s)}=$ $\frac{\mathcal{C}_{e, i-1}(s) \mathcal{G}_{i-2}(s)+\mathcal{D}_{e, i-1}(s)}{\mathcal{C}_{e, i}(s) \mathcal{G}_{i-1}(s)+\mathcal{D}_{e, i}(s)}$,in the case of general heterogeneous platoons.

In the following we examine the worst situation when an LPF-ASP vehicle starts following its predecessor and a distant vehicle, and these two move independently of each other, i.e., the platoon is actually broken. Suppose without loss in generality that vehicle 1 is the true leader instead of vehicle 0 , and vehicles $2,3, \ldots$ are characterized by (16)-(18). For LPF vehicles the "leader" information $a_{0}$ is disturbing. It will be shown that, for LPF-ASP vehicles, this disturbance imposes only a finite spacing error that depends on the speed difference of vehicle 0 and the immediate predecessor $i-1$.

Let us introduce the following notation

$$
a_{i}(s)=\mathcal{H}_{i}(s) a_{1}(s)+\mathcal{M}_{i}(s) a_{0}(s), \quad i \geq 2,
$$

for describing the acceleration of vehicle $i$ in terms of the motion of the true leader and the misleader. Both $\mathcal{H}_{i}$ and $\mathcal{M}_{i}$ are stable SISO systems and evolve with vehicle index as follows,

$$
\begin{aligned}
\mathcal{H}_{i}(s) & =\mathcal{A}_{i}(s) \mathcal{H}_{i-1}(s), & \mathcal{H}_{2}(s) & =\mathcal{A}_{2}(s) \\
\mathcal{M}_{i}(s) & =\mathcal{A}_{i}(s) \mathcal{M}_{i-1}(s)+\mathcal{B}_{i}(s), & \mathcal{M}_{2}(s) & =\mathcal{B}_{2}(s) .
\end{aligned}
$$

It follows from (44) and (45) that the broken platoon is heterogeneous string stable with respect to $a_{i}$ in the $\mathcal{L}_{2}$ norm if and only if the unbroken platoon (39) is heterogeneous string stable with respect to $a_{i}$ in the $\mathcal{L}_{2}$ norm. Concerning spacing errors, the following theorem holds.

Theorem 5: Suppose that $a_{0}, a_{1} \in \mathcal{B L}_{2, \infty}(\delta, c)$. Suppose that the platoon (43)-(45) is heterogeneous string stable with respect to $a_{i}$ in the $\mathcal{L}_{2}$ norm, i.e., the sequence $\left\|a_{i}\right\|_{2}$ is uniformly bounded. Assume that for all $i \geq 2 \mathcal{H}_{i}(s)$ is of type $k>0$ and $\mathcal{M}_{i}(s)$ has at least one zero at $s=0$. If for each vehicle both $\mathcal{C}_{e, i}(s)$ and $\mathcal{D}_{e, i}(s)$ have at most one integrators, but the other poles are stable, then the platoon is ultimately heterogeneous string stable with respect to spacing error $e_{i, i-1}$ in the sense of Definition 3.

Proof. Introduce the following transfer function: $W_{i-1}(s) \triangleq$ $\frac{1}{s^{k}}\left(1-\mathcal{H}_{i-1}(s)\right)$, which is stable and uniformly bounded by assumption. From (18) and (43) the spacing error can be written as (40), where $e_{i, i-1}^{\left(\mathcal{L}_{\infty}\right)}(s)=\mathcal{C}_{e, i}(s) a_{1}(s)+\mathcal{D}_{e, i}(s) a_{0}(s)$ and $e_{i, i-1}^{\left(\mathcal{L}_{2}\right)}(s)=\mathcal{C}_{e, i}(s) \mathcal{M}_{i-1}(s) a_{0}(s)-s^{k} \mathcal{C}_{e, i}(s) W_{i-1}(s) a_{1}(s)$. By the conditions of the theorem $e_{i, i-1}^{\left(\mathcal{L}_{2}\right)} \in \mathcal{L}_{2}$ and $e_{i, i-1}^{\left(\mathcal{L}_{\infty}\right)} \in \mathcal{L}_{\infty}$ and the proof continues as in the proof of Theorem 4.

An LPF-ASP vehicle satisfies the conditions of the theorem which implies that even if it connects, by a mistake, to a distant vehicle, which is actually not related to the rest of the platoon, the spacing error remains bounded in the sense of Definition 3. Since LPF-ASP vehicles satisfy also that $\mathcal{C}_{e, i}+\mathcal{D}_{e, i}$ is stable, the finite $\mathcal{L}_{\infty}$ term of the spacing error reduces to $e_{i, i-1}^{\left(\mathcal{L}_{\infty}\right)}(s)=$ $\mathcal{D}_{e, i}(s)\left(a_{0}(s)-a_{1}(s)\right)=\tilde{\mathcal{D}}_{e, i}(s)\left(v_{0}(s)-v_{1}(s)\right)$, where $\tilde{\mathcal{D}}_{e, i}=$ $\frac{1}{s} \mathcal{D}_{e, i}(s)$ is stable. It follows that the $\mathcal{L}_{\infty}$ part of the spacing error is bounded by the induced peak-to-peak gain of $\tilde{\mathcal{D}}_{e, i}$ times the peak value of the speed difference $\left|v_{0}(t)-v_{1}(t)\right|$.

\section{LPF-ASP DESIGN CONSIDERATIONS}

The goal of the design is to find the parameters for the VP model and the LPF controller such that string stability criteria are satisfied, control input is realizable and spacing errors are as small as possible. Furthermore, the system must have sufficient tolerance for noise, disturbances and modeling uncertainties, which are not considered in this paper. A systematic synthesis procedure for this multi-criterion and non-convex optimization problem is still missing. We have to content ourself with a simple heuristic design method that is summarized in the following steps.

1) The following design parameters are initialized: $\tau_{i}^{v}:=$ $\tau_{i}, \omega_{1, i}^{v}=\frac{1}{\tau_{i}^{v}}, \omega_{2, i}^{v}=\frac{1}{\tau_{i}^{v}}, \omega_{3, i}^{v}=\frac{1}{\tau_{i}}, \rho_{a, i}=\rho_{v, i}=$ $\rho_{p, i}=0.5$.

2) Parameters $K_{a, i}^{v}, K_{v, i}^{v}$ and $K_{p, i}^{v}$ are determined such that the feedback loop for VP model (22)-(23) is stabilized 
TABLE I

PARAMETERS OF LPF-ASP VEHICLE $i$

\begin{tabular}{|l||l|}
\hline$\tau_{i}=0.5$ & time-constant of the vehicle dynamics \\
\hline$\tau_{i}^{v}=0.5$ & time-constant of the VP model \\
\hline$K_{a, i}^{v}=0.995$ & \\
$K_{v, i}^{v}=2.189$ & PF control parameters for the VP model \\
$K_{p, i}^{v}=0.398$ & \\
\hline$C_{a, i}=2.5$ & reference tracking controller parameters \\
$C_{v, i}=5.5$ & in the virtual spacing policy estimator \\
$C_{p, i}=1$ & \\
\hline$K_{a, i}^{0}=K_{a, i}^{i-1}=0.4975$ & fixed LPF controller parameters \\
$K_{v, i}^{0}=K_{v, i}^{i-1}=1.0945$ & \\
\hline$K_{p, i}^{0}=\rho_{p, i} 0.398$ & LPF controller parameters \\
$K_{p, i}^{i-1}=\left(1-\rho_{p, i}\right) 0.398$ & parameterized by $\rho_{p, i}$ \\
\hline$R_{i, i-1}(t)=10$ & Constant spacing policy w.r.t. predecessor [m] \\
\hline
\end{tabular}

with phase margin close to $90^{\circ}$ and desired bandwidth $\omega_{1, i}^{v}$.

3) Step 2) is repeated for the reference tracking loop (31) to obtain $C_{a, i}, C_{v, i}$ and $C_{p, i}$ with desired bandwidth $\omega_{2, i}^{v}$.

4) The design of LPF controller (2) begins with a predecessor following controller designed by using the method of Step 2) for the plant $H_{i}(s)$ with desired bandwidth $\omega_{3, i}^{v}$. Let the obtained parameters be denoted by $K_{a, i}$, $K_{v, i}$ and $K_{p, i}$, and finally let

$$
\begin{array}{ll}
K_{a, i}^{0}:=\rho_{a, i} K_{a, i}, & K_{a, i}^{i-1}:=\left(1-\rho_{a, i}\right) K_{a, i}, \\
K_{v, i}^{0}:=\rho_{v, i} K_{v, i}, & K_{v, i}^{i-1}:=\left(1-\rho_{v, i}\right) K_{v, i}, \\
K_{p, i}^{0}:=\rho_{p, i} K_{p, i}, & K_{p, i}^{i-1}:=\left(1-\rho_{p, i}\right) K_{p, i} .
\end{array}
$$

5) Evaluate the criteria and modify the design parameters if necessary (a numeric optimization procedure can also be applied).

The advantage of the above simple parametrization is that some fundamental relationships between string stability, spacing performance and some basic properties of the three feedback-loops can be experienced. In the following section the effect of parameter $\rho_{p, i}$ is analyzed in detail, since $\rho_{p, i}$ is a weighting factor for the position information received from the leader in LPF control structures. Results that support the usefulness of applying $\rho_{p, i}>0$ justify the problem setting of this paper.

\section{NumeriCAl ANALYSis AND Simulations}

In this section we analyze string stability of the proposed LPF-ASP controller with respect to acceleration, control effort and spacing error through numerical examples. We examine also the significance of leader position information. An LPFASP vehicle (32)-(35) is designed as described in Section V. The parameters are given in Table I.

1) String Stability Analysis: Two parameters, namely $K_{p, i}^{0}$ and $K_{p, i}^{i-1}$, which are the gains for the position feedback regarding the leader and the predecessor, respectively, are parameterized by a weighting factor $\rho_{p, i} \in[0,1]$,

$$
K_{p, i}^{0}=\rho_{p, i} 0.398, \quad K_{p, i}^{i-1}=\left(1-\rho_{p, i}\right) 0.398 .
$$

The choice $\rho_{p}=0$ covers the case when no leader's position information is utilized in the control. Increasing $\rho_{p}$ represent the increasing significance of this information.
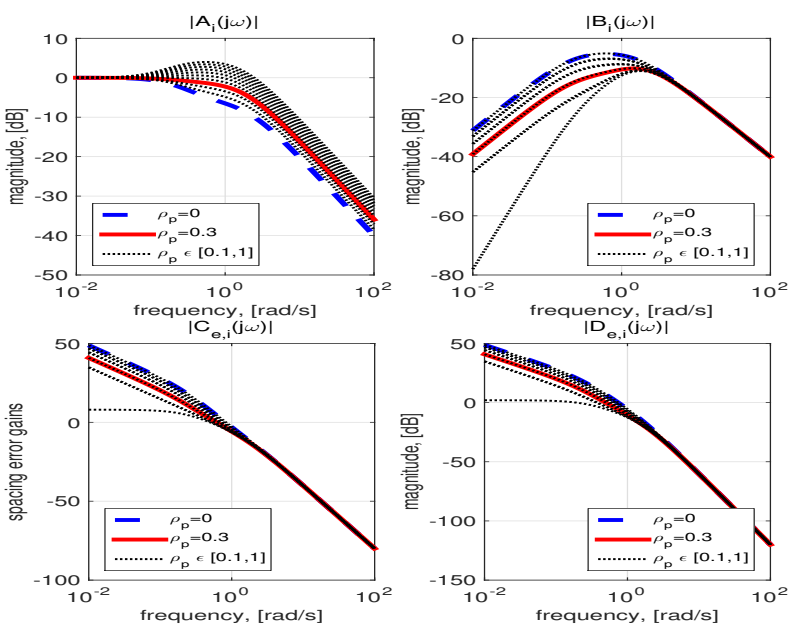

Fig. 5. Magnitude plots of transfer functions $\mathcal{A}_{i}, \mathcal{B}_{i}, \mathcal{C}_{e, i}$ and $\mathcal{D}_{e, i}$ for an LPF-ASP vehicle.

It can be shown that the type 2 control structure of LPFASP architectures ensures $\mathcal{B}_{i}(j 0)=0$ and $\mathcal{A}_{i}(j 0)=1$ for any choice of the LPF parameters. It can be seen in Fig. 5 that the gain $\left|\mathcal{A}_{i}(j \omega)\right|$ increases for $\omega>0$ with increased weight, $\rho_{p, i}$ and $\rho_{p, i}>0.4$ results in string instability. As a conclusion, omitting feedback from the leader position seems to be reasonable as far as string stability (with respect to accelerations) is concerned. On the other hand, each of $\mathcal{C}_{e, i}$ and $\mathcal{D}_{e, i}$ contain one integrator and the gains decrease with increasing $\rho_{p, i}$, which suggest that spacing errors can be decreased if leader position information is utilized in the control.

2) Simulation Examples: The situation in which the ASP approach can be useful is imagined as follows. Suppose our vehicle is equipped with PF-CTHSP control functionality so it can follow any vehicle with a safe headway-time. If an ad hoc $\mathrm{V} 2 \mathrm{~V}$ communication network is present and a vehicle is detected in the lane ahead with the ability of sending kinetic information, our vehicle starts computing the virtual spacing policy of its predecessor with respect to that distant vehicle. The behavior of the "platoon" between the two vehicles is classified and a decision is made whether to utilize the information of the distant vehicle. If the answer is positive, the PF-CTHSP controller is gradually changed into an LPF-ASP controller, and the headway parameter, $h_{i, i-1}$, is continuously decreased to zero. As a result, the LPF-ASP vehicle follows its predecessor safely within a short gap. Details on the decision process mentioned above is still to be worked out.

The following time-domain simulations show 1) string stability with respect to spacing errors; 2) that leader position information allows for shorter and smaller spacing errors as compared with the case of $\rho_{p, i}=0$ (no leader position feedback); and 3) behavior of LPF-ASP vehicles when the platoon is suddenly breaks up.

In the first example the leader accelerates by $1 \mathrm{~m} / \mathrm{s}^{2}$ for $t \in$ $[0,5 s]$. The leader is followed by ten PF-CTHSP vehicles with $h_{j, j-1}=1 s, j=1,2, \ldots, 10$. They are followed by $90 \mathrm{LPF}-$ ASP vehicles with parameters given in Table I. The platoon starts from standstill with initially zero spacing errors. It is emphasized that LPF-ASP vehicles keep constant distances 

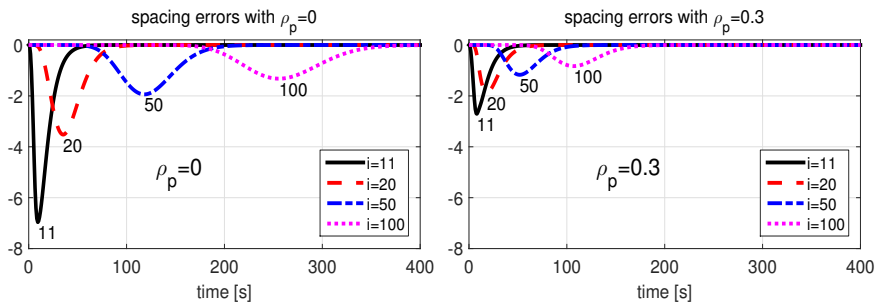

Fig. 6. Spacing errors of selected LPF-ASP vehicles without $\left(\rho_{p}=0\right.$ - left), and with position feedforward ( $\rho_{p}=0.3$ - right).

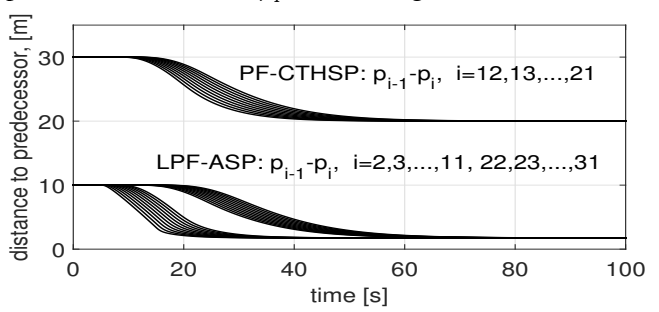

Fig. 7. Broken platoon experiment. Speed of vehicle 1 drops from $20 \mathrm{~m} / \mathrm{s}$ to $10 \mathrm{~m} / \mathrm{s}$ with deceleration of $-1 \mathrm{~m} / \mathrm{s}^{2}$ while $v_{0}(t)=20 \mathrm{~m} / \mathrm{s}$. Vehicle 1 is followed by 10 LPF-ASP, 10 PF-CTHSP and again 10 LPF-ASP vehicles.

with respect to their predecessors, which are much smaller as compared with the speed dependent gaps of the PF-CTHSP vehicles. The spacing errors of vehicles $i \in\{11,20,50,100\}$ are shown in Fig. 6 for two cases with $\rho_{p, i}=0.3$ and $\rho_{p, i}=0$, respectively. The length of the transients in the spacing errors was doubled, the peak of the spacing errors were more than doubled in the case when no leader position information was utilized, which shows the advantages of position feedforward from the leader.

In the second example all vehicles are traveling with speed of $20 \mathrm{~m} / \mathrm{s}$, initially. Then at time $t=5 s$ vehicle 1 decelerates with $-1 \mathrm{~m} / \mathrm{s}^{2}$ to speed $v_{1}(t)=10 \mathrm{~m} / \mathrm{s}, t>15 \mathrm{~s}$, while the leader keeps its speed, $v_{0}(t)=20 \mathrm{~m} / \mathrm{s}$. Vehicle 1 (may be a human driven vehicle) is followed by ten LPF-ASP vehicles, $i=2,3, \ldots, 11$, ten PF-CTHSP vehicles, $i=12,13, \ldots, 21$, and again ten LPF-ASP vehicles, $i=22,23, \ldots, 31$. Fig. 7 shows the distances between the vehicles. It can be seen that, according to Definition 3, the spacing errors of the LPF-ASP vehicles remain ultimately bounded, and also the transient term $e_{i, i-1}^{\left(\mathcal{L}_{2}\right)}$ has small peak values. It is shown in Section IV-E that the $\mathcal{L}_{\infty}$ part of the spacing error is proportional to the peakto-peak gain of $\tilde{\mathcal{D}}_{e, i}=\frac{1}{s} \mathcal{D}_{e, i}(s)$. Therefore, one goal of the control design should be to minimize this gain, if LPF-ASP control is intended to be applied in completely unorganized platoons.

\section{CONCLUSION}

We have addressed the problem of heterogeneous spacing policies in multi-brand, ad hoc platoons and demonstrated that utilizing relative motion information from a leader vehicle leads to collision of vehicles, when the consistency of spacing policies are not ensured on-line. To resolve the problem we have proposed a leader and predecessor following (LPF) control strategy where the spacing policy with respect to the leader was adapted (ASP) based on a virtual predecessor vehicle model. Conditions for string stability of platoons consisting of LPF-ASP vehicles has been derived based on a recursive description of the platoon. We have shown by numerical analysis that string stability with respect to acceleration and control effort can be guaranteed by appropriately choosing the LPF-ASP control parameters, while the bounds on the spacing errors depend on the relative motion of the leader and the predecessor vehicles. It has been shown that smaller spacing errors and shorter transients can be achieved with the proposed adaptive spacing policy when relative position information with respect to the leader is utilized in the control.

Future work should elaborate analysis and synthesis techniques to address the issue of robustness to modeling uncertainties in the vehicle dynamics, disturbances, sensor noise, initial conditions, and the trade-off between control effort, string stability and worst-case spacing errors.

\section{REFERENCES}

[1] S. E. Li, Y. Zheng, K. Li, and J. Wang, "An overview of vehicular platoon control under the four-component framework," in 2015 IEEE Intelligent Vehicles Symposium (IV), June 2015, pp. 286-291.

[2] D. Swaroop and J. Hedrick, "String stability of interconnected systems," Automatic Control, IEEE Transactions on, vol. 41, no. 3, pp. 349-357, Mar 1996

[3] D. Swaroop, "String stability of interconnected systems: An application to platooning in automated highway systems," PhD dissertation, 1994.

[4] G. Orosz, R. E. Wilson, and G. Stépán, "Traffic jams: dynamics and control," Philosophical Transactions of the Royal Society of London A: Mathematical, Physical and Engineering Sciences, vol. 368, no. 1928, pp. 4455-4479, 2010.

[5] L. Alvarez and R. Horowitz, "Safe platooning in automated highway systems," PATH Research Report, UCB-ITS-PRR-97-46, 1997.

[6] EU Comission Funding Opportunities, "Topic: Multibrand platooning in real traffic conditions. Topic identifier: Art-03-2017," 2015. [Online]. Available: https://ec.europa.eu/research/participants/portal/desktop/en/opportunities /h2020/topics/art-03-2017.html

[7] G. Silberg and R. Wallace, "Self-driving cars: The next revolution," Center for Automotive Research, Transportation Systems Analysis Group, Tech. Rep., 2012.

[8] S. Öncü, N. van de Wouw, and H. Nijmeijer, "Cooperative adaptive cruise control: Tradeoffs between control and network specifications," in 14th International IEEE Conference on Intelligent Transportation Systems (ITSC), 2011, pp. $2051-2056$.

[9] G. Naus, R. Vugts, J. Ploeg, R. van de Molengraft, and M. Steinbuch, "Cooperative adaptive cruise control, design and experiments," Proc. 2010 American Control Conference, Baltimore, MD, pp. 6145-6150, 2010.

[10] S. E. Shladover, C. Nowakowski, D. Cody, F. Bu, J. O’Connell, J. Spring, S. Dickey, and D. Nelson, "Effects of cooperative adaptive cruise control on traffic flow: Testing drivers' choices of following distances," PATH Research Report UCB-ITS-PRR-2009-23, 2009.

[11] U. Montanaro, M. Tufo, G. Fiengo, M. di Bernardo, and S. Santini, "On convergence and robustness of the extended cooperative cruise control," in 53rd IEEE Conference on Decision and Control, Dec 2014, pp. 40834088.

[12] G. Rödönyi and Z. Szabó, "Adaptation of spacing policy of autonomous vehicles based on an unknown input and state observer for a virtual predecessor vehicle," in 2016 IEEE 55th Conference on Decision and Control (CDC), Dec 2016, pp. 1715-1720.

[13] J. I. Ge, S. S. Avedisov, and G. Orosz, "Stability of connected vehicle platoons with delayed acceleration feedback," Proceedings of the ASME 2013 Dynamic Systems and Control Conference, DSCC2013, pp. 1-10, 2013.

[14] J. I. Ge and G. Orosz, "Dynamics of connected vehicle systems with delayed acceleration feedback," Transportation Research Part C: Emerging Technologies, vol. 46, no. 0, pp. 46 - 64, 2014.

[15] A. Geiger, M. Lauer, F. Moosmann, B. Ranft, H. Rapp, C. Stiller, and J. Ziegler, "Team annieway's entry to the 2011 grand cooperative driving challenge," IEEE Transactions on Intelligent Transportation Systems, vol. 13, no. 3, pp. 1008-1017, Sept 2012.

[16] K. Lidstrom, K. Sjoberg, U. Holmberg, J. Andersson, F. Bergh, M. Bjade, and S. Mak, "A modular cacc system integration and design," IEEE Transactions on Intelligent Transportation Systems, vol. 13, no. 3, pp. 1050-1061, Sept 2012. 
[17] J. Tóth and G. Rödönyi, "String stability preserving adaptive spacing policy for handling saturation in heterogeneous vehicle platoons," IFAC 2017 World Congress, Toulouse, France, pp. 8855-8860, 2017.

[18] Y. Zheng, S. E. Li, J. Wang, D. Cao, and K. Li, "Stability and scalability of homogeneous vehicular platoon: Study on the influence of information flow topologies," IEEE Transactions on Intelligent Transportation Systems, vol. 17, no. 1, pp. 14-26, Jan 2016.

[19] Y. Zheng, S. E. Li, K. Li, and L. Y. Wang, "Stability margin improvement of vehicular platoon considering undirected topology and asymmetric control," IEEE Transactions on Control Systems Technology, vol. 24, no. 4, pp. 1253-1265, July 2016.

[20] J. Ploeg, N. van de Wouw, and H. Nijmeijer, " $\mathcal{L}_{p}$ string stability of cascaded systems: Application to vehicle platooning," Control Systems Technology, IEEE Transactions on, vol. 22, no. 2, pp. 786-793, March 2014.

[21] E. Shaw and J. Hedrick, "String stability analysis for heterogeneous vehicle strings," in American Control Conference, 2007. ACC '07, 2007, pp. 3118-3125.

[22] H. Köroğlu and P. Falcone, "Robust static output feedback synthesis for platoons under leader and predecessor feedback," International Journal of Robust and Nonlinear Control, pp. n/a-n/a, 2016.

[23] D. Yanakiev and I. Kanellakopoulos, "Nonlinear spacing policies for automated heavy-duty vehicles," IEEE Transactions on Vehicular Technology, vol. 47, no. 4, pp. 1365-1377, Nov 1998.

[24] J. Ploeg, N. van de Wouw, and H. Nijmeijer, "Fault tolerance of cooperative vehicle platoons subject to communication delay," 12th IFAC Workshop on Time Delay Systems, TDS 2015 - Ann Arbor, Michigan, USA, vol. 48, no. 12, pp. 352 - 357, 2015.

[25] J.-S. Yang and W. S. Levine, "Specification of control systems," in The Control Handbook, W. S. Levine, Ed. CRC Press INC, 1999, ch. 10.1, p. 160.

[26] A. Pant, P. Seiler, and K. Hedrick, "Mesh stability of look-ahead interconnected systems," Automatic Control, IEEE Transactions on, vol. 47, no. 2, pp. 403-407, Feb 2002.

[27] G. Rödönyi, P. Gáspár, J. Bokor, and L. Palkovics, "Experimental verification of robustness in a semi-autonomous heavy vehicle platoon," Control Engineering Practice, vol. 28, pp. 13 - 25, 2014.

[28] G. Rödönyi, "Leader and predecessor following robust controller synthesis for string stable heterogeneous vehicle platoons," in 8th IFAC Symposium on Robust Control Design, ROCOND, Bratislava, Slovak Republic, 2015, pp. 154-159.

[29] H. Lin and P. J. Antsaklis, "Stability and stabilizability of switched linear systems: A survey of recent results," IEEE Transactions on Automatic Control, vol. 54, no. 2, pp. 308-322, Feb 2009.

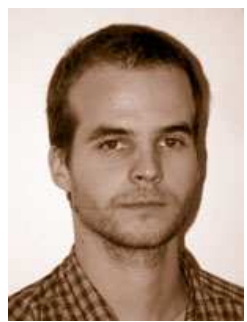

Gábor Rödönyi received his Ph.D. degree from the Faculty of Electrical Engineering, Budapest University of Technology and Economics in 2010. He is a research fellow in the Systems and Control Laboratory of the Institute for Computer Science and Control, Hungarian Academy of Sciences. His research interests include interconnected systems, control of vehicle platoons, linear parameter-varying systems, robust control, identification for control. Email: rodonyi@sztaki.hu 